\author{
Military Technical \\ College \\ Kobry El-Kobba \\ Cairo, Egypt
}

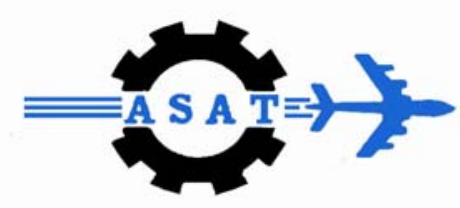

12-th International

Conference on

Aerospace Sciences \&

Aviation Technology

\title{
JAMMING OF MULTICARRIER FREQUENCY HOPPING COHERENT BPSK SYSTEM OVER AWGN CHANNEL
}

\author{
EL-MAHDY* A. E., KHAIRY* E. B. and SAMY* A.M.,
}

\begin{abstract}
Most frequency hopping $(\mathrm{FH})$ systems use non-coherent reception due to the difficulty of synchronization of the carrier phase. This implies a performance loss in these systems. Multicarrier frequency hopping systems make coherent reception feasible. The performance of these systems in presence of jamming is seldom investigated. In this paper, the performance of coherent multicarrier frequency hopping binary PSK (MC-FH/BPSK) system under the effect of jamming is studied. The considered types of jamming are partial band jamming and broadband jamming. It is shown that a smart jammer should be present in all subbands. The error performance of the MC-FH/BPSK system is compared with that one of the single carrier FH/BPSK system in presence of jamming. Computer simulations are provided to validate the theoretical developments.
\end{abstract}

\section{KEY WORDS}

Multicarrier system, frequency hopping, and jamming.

\footnotetext{
* Egyptian Armed Forces
} 


\section{INTRODUCTION}

Over the past few decades frequency hopping spread spectrum (FH-SS) communication systems have attracted great interest for combating intentional and unintentional jamming. However, the $\mathrm{FH}-\mathrm{SS}$ system is vulnerable to band-limited jamming because the jammer bandwidth can be adjusted to a value that maximizes the error probability of the communicator system [1], [2]. Partial band noise jamming (PBJ) is an effective jamming method against FH-SS systems [3]. However, an efficient countermeasure to PBJ for the communicator is then to use diversity. Traditionally, diversity was obtained via multiple hops per information (or coded) symbol. As investigated in [1] and [2], such a fast hopping makes difficulty to synchronize the carrier phase and, consequently, imposes the use of a noncoherent receivers. Thus, a significant loss in error performance is presented, due to both noncoherent demodulation and noncoherent combining of the received diversity replicas. Some authors have studied a solution that makes coherent reception feasible such as [4], and [5]. The idea of their solution is to maintain a continuous phase at the transmitter when the carrier is hopping. This makes carrier synchronization at the receiver easier. A major problem of this solution is that, phase continuity cannot be maintained for large spectrum spreading. But phase continuity is technically possible if the bandwidth, inside which the signal is hopping, is sufficiently small. Another solution to this problem is to use MC-FH systems. In these systems, the frequency band is partitioned into $L$ disjoint subbands on which $L$ replicas of the signal are simultaneously transmitted. In these systems coherent reception is easy to implement [6]. Moreover, if side information on the jammer and noise level is available, optimum coherent combining results in a large improvement in error performance as compared to single carrier $\mathrm{FH}-\mathrm{SS}$ system. MC-FH satisfies this requirement, and thus allows one to obtain coherent reception.

In this paper, the performance of MC-FH/BPSK system is studied in presence of both partial band and broadband jamming. The error performance of this system is compared with that one of the single carrier FH/BPSK system under the effect of jamming. The paper is organized as follows. The system model is described in section 2. The bit error probability of the system under partial band jamming is derived in section 3. The worst case jamming is also discussed in this section. Moreover, the performance of the system under broadband jamming is analyzed. Simulation results is then given in section 4. Conclusions are provided in section 5.

\section{SYSTEM MODEL}

Transmitter and receiver block diagrams of MC-FH system with coherent BPSK are shown in Fig. 1 and Fig. 2 respectively. The information symbols, $\left\{a_{k}\right\}$, are transmitted with a rate $1 / T_{b}, a_{k} \in\{-1,+1\}$ and $k$ is the time index, $k=\{0,1, \ldots$.$\} . The replicas of$ these bits are transmitted simultaneously over the $L$ subbands. For simplicity sake, it is assumed that there is one frequency hop per signaling interval $T_{b}$. Let $W_{S S}$ be the total communicator bandwidth. Then the bandwidth of a subband, $W_{s b}$, is equal to $W_{S S} / L$. The bit $a_{k}$ is sent on $L$ branches, the replica of $a_{k}$ on branch $l \in\{1, \ldots \ldots, L\}$ is 
denoted $a_{k, l}$. Each replica is transmitted using a (BPSK) modulation which occupies a frequency interval that is designated by subchannels. Obviously a subchannel is a subset of a subband. It is assumed that each subband contains $h=N / L$ different carrier frequencies spaced apart by $f_{d}$. The total number of carriers that can be accommodated in the communication band is $N$. Thus the number of carriers in a subband is an integer $N / L$. It is clear that $h$ is the number of available hops for a symbol replica $a_{k, l}$. $f_{d}$ is chosen such that each pair of carriers is orthogonal i.e. $f_{d}=1 / T_{b}$, where $T_{b}$ is the bit time interval. The frequency hopped signal is given by:

$$
s(t)=\sum_{k} \sum_{l=1}^{L} \sqrt{2 S} a_{k, l} \exp \left[j 2 \pi f_{l}\left(t-k T_{b}\right)\right] P_{T_{b}}\left(t-k T_{b}\right)
$$

where $f_{l}$ is a carrier frequency in subband $l,\left\{a_{k, l}\right\}$ is binary sequence, and $P_{T_{b}}(t)$ is a unit rectangular pulse over interval $T_{b}$ which satisfies the orthogonality condition

$$
\int_{-\infty}^{\infty} P_{T_{b}}\left(t-m T_{b}\right) P_{T_{b}}\left(t-n T_{b}\right) d t=\delta_{m, n}\left\|P_{T_{b}}\right\|^{2}, \text { where } \delta_{m, n}= \begin{cases}1 & \text { if } m=n \\ 0 & \text { otherwise }\end{cases}
$$

Note that, with the above assumptions, the hopper is different for each subband $l$. From (1) it is clear that the total transmitted power is equal to $L S$ where $S$ is the transmitted power for one subband $l$. The channel is assumed to be AWGN channel, then the received signal is given by

$$
r(t)=s(t)+n(t)
$$

where $n(t)$ is a zero mean complex Gaussian noise process, independent of signal and with power spectral density (psd) $2 N_{0}$. The block diagram of the MC-FH/BPSK receiver is shown in Fig.2. To separate the signals of different carriers, $L$ branches are employed. Each branch contains a dehopper followed by a correlator. The dehopper of each branch knows the hopping pattern in that branch. The real part of the sum of the correlators outputs, $Z_{k, m}$, of all subbands gives the decision variable $Z_{k}$ which is then compared to zero threshold to make a decision about the information bit corresponding to that interval.

\section{PARTIAL BAND JAMMING}

The jamming signal, denoted $J(t)$, is a stationary zero mean complex Gaussian process active on a fraction $\eta$ of the signal bandwidth. When the bandpass jammer is present on a hopping subchannel, it is assumed that it has a flat power spectral density $J_{0} / 2$. When the jammer is present in a subband, it covers a fraction $v$ of its bandwidth $W_{s b}$. Let $M$ be the set of subbands where the jammer is present and 
$M \leq L$, then $\eta=v M / L$. If the jammer is present in all subbands then $M=L$ and consequently $\eta=v$.

This section is divided into four subsections. In subsection 3.1, theoretical performance evaluation of the MC-FH/BPSK system in presence of partial band jamming is derived. In subsection 3.2, the worst case jamming state is obtained. In subsection 3.3, a comparison between the performance of MC-FH/BPSK and single carrier FH/BPSK under the effect of partial band jamming is investigated. In subsection 3.4, the performance of MC-FH/BPSK system in presence of broadband jamming is provided. Moreover the performance comparison between the MC$\mathrm{FH} / \mathrm{BPSK}$ and single carrier is also presented.

\subsection{Theoretical Performance Evaluation.}

In this subsection, the bit error probability (BEP) of the MC-FH/BPSK system is evaluated under partial band jamming. Without loss of generality, the received signal is considered in the interval number zero. In this interval the received jammed signal is given by

$$
r(t)=\sum_{l=1}^{L} a_{0, l} \sqrt{2 S} \exp j 2 \pi f_{l} t+n(t)+J(t)
$$

where $J(t)$ is the jamming signal. Consequently the output of the $i^{\text {th }}$ dehopper, $q_{i}(t)$, is given by

$$
\begin{aligned}
q_{i}(t) & =r(t) \exp \left(-j 2 \pi f_{i} t\right) \\
& =\sum_{l=1}^{L} a_{0, I} \sqrt{2 S} \exp j 2 \pi\left(f_{l}-f_{i}\right) t+n(t) \exp \left(-j 2 \pi f_{i} t\right)+J(t) \exp \left(-j 2 \pi f_{i} t\right)
\end{aligned}
$$

The output of the $i^{\text {th }}$ subband correlator, $Z_{0, i}$, is given by

$$
\begin{aligned}
Z_{0, i}= & \frac{1}{T_{b}} \int_{0}^{T_{b}} \sum_{l=1}^{L} a_{0, l} \sqrt{2 S} \exp \left[j 2 \pi\left(f_{l}-f_{i}\right) t\right] d t+\frac{1}{T_{b}} \int_{0}^{T_{b}} n(t) \exp \left(-j 2 \pi f_{i} t\right) d t \\
& +\frac{1}{T_{b}} \int_{0}^{T_{b}} J(t) \exp \left(-j 2 \pi f_{i} t\right) d t
\end{aligned}
$$

because of different carriers are orthogonal, the integral in the first term of (6) is nonzero only for $l=i$ otherwise it equals to zero. Assuming that the transmitted bit at the interval number zero, $a_{0, l}$, is equal to, 1 , where $1 \leq l \leq L$, consequently we have:

$$
Z_{0, i}=\sqrt{2 S}+n_{i}+J_{i}
$$


Where $n_{i}$ is the complex noise component at the $i^{\text {th }}$ subband correlator and it is given by:

$$
n_{i}=\frac{1}{T_{b}} \int_{0}^{T_{b}} n(t) \exp \left(-j 2 \pi f_{i} t\right) d t
$$

and, $J_{i}$ is the complex jamming component at the $i^{\text {th }}$ subband correlator output that is given by:

$$
J_{i}=\frac{1}{T_{b}} \int_{0}^{T_{b}} J(t) \exp \left(-j 2 \pi f_{i} t\right) d t
$$

The jamming power appears as an additional additive Gaussian noise source in each of the $M$ jamming subbands with power spectral density $J_{0}=J_{a v} / \eta W_{s s}$, where $J_{a v}$ is the total average jamming power. Assume that $m$ out of $M$ replicas are hit by the jammer, then the decision variable $Z_{k}$ is equal to

$$
Z_{k}=\operatorname{Re}\left\{\sum_{i=1}^{L} Z_{0, i}\right\}=L \sqrt{2 S}+N+J
$$

where $N=\sum_{i=1}^{L} \operatorname{Re}\left(n_{i}\right)$, and $J=\sum_{i=1}^{L} \operatorname{Re}\left(J_{i}\right) \cdot \ln (10), N$ is the total noise component and $J$ is the total jamming component caused by the partial band jammer. To obtain the distribution of the random variable $Z_{k}$, it is necessary to obtain the distribution of both $N$ and $J$. Again, as the carriers are orthogonal, the random variables $n_{i}^{s}$ are independent and identical distribution (i.i.d.) with Gaussian distribution. Consequently, $N$ is a Gaussian random variable (r.v.) with zero mean and variance $\sigma_{N}^{2}=\frac{N_{0} L}{T_{b}}$. Similarly, $J$ is a Gaussian r.v. with zero mean and variance $\sigma_{J}^{2}=\frac{J_{0} m}{T_{b}}$. Since the noise and the jamming are independent, the variance of $(N+J)$ is equal to the sum of their variances and it is given by

$$
\sigma_{T}^{2}=\frac{N_{0} L+J_{0} m}{T_{b}}
$$

Consequently the pdf of $Z_{k}$, conditioned on the transmitted bit is one, is given by

$$
f_{Z_{k} / 1}\left(z_{k}\right)=\frac{1}{\sqrt{2 \pi \sigma_{T}^{2}}} \exp \left(\frac{-\left(z_{k}-L \sqrt{2 S}\right)^{2}}{2 \sigma_{T}^{2}}\right)
$$


Note that the decision variable $Z_{k}$ is compared with zero threshold to decide between 1 and -1 . Then the bit error probability (BEP) is equal to the probability that the decision variable is less than zero given that the transmitted bit is one. Then the conditional BEP, $P(e / m)$, under partial band jamming can be written as

$$
P(e / m)=\int_{-\infty}^{0} f_{z_{k} / 1}\left(z_{k}\right) d z_{k}=Q\left(\sqrt{\frac{2 E_{b}}{N_{0}+(m / L) J_{0}}}\right)
$$

where $E_{b}$ is the energy per bit for the MC-FH/BPSK system which is given by $E_{b}=L S T_{b}$. From (13), it is clear that as $m$ increases, the degradation in system performance increases. Then, the probability that $m$ out of $M$ replicas are hit simultaneously by the jammer is given by

$$
P_{m}=\left(\begin{array}{l}
M \\
m
\end{array}\right) v^{m}(1-v)^{M-m}
$$

There are $(L-M)$ replicas that are never hit by the jammer. Therefore the bit error probability, $P_{e}$, of the system is given by

$$
P_{e}=\sum_{m=0}^{M} P_{m} P(e / m)=\sum_{m=0}^{M}\left(\begin{array}{l}
M \\
m
\end{array}\right) v^{m}(1-v)^{M-m} Q\left(\sqrt{\frac{2 E_{b}}{N_{0}+\frac{m}{L} J_{0}}}\right)
$$

\subsection{Worst Case Jamming.}

In MC system, worst case jamming happens when $M=L$, that is, the jammer is present in all subbands. If $M<L$ i.e. $\exists$ one subband or more without jamming this implies that when $E_{b} / N_{0}$ tends to infinity, $P_{e}$ tends to zero, independent of the jamming power. This causes replicas of $a_{k}$ which is found in the unjammed subbands are detected correctly. When $M=L$, then $\eta=v$ and knowing that $J_{0}=N_{j} / \eta$ the BEP given by (15) becomes

$$
P_{e}=\sum_{m=0}^{L}\left(\begin{array}{c}
L \\
m
\end{array}\right) \eta^{m}(1-\eta)^{L-m} Q\left(\sqrt{\frac{2 E_{b}}{N_{0}+\frac{m}{L}\left(N_{j} / \eta\right)}}\right)
$$

where $N_{j}$ is the jamming power spectral density. Recall that a smart jammer should be present in all subbands. However, according to the error probability given in (16), the jammer can select its parameter $\eta$ so that the communicator BEP is maximized. 
The plot of the exact BEP given by (16) versus $E_{b} / N_{j}$ is illustrated in Fig.3. The figure is plotted for different values of $\eta$, with $L=8$, and with $E_{b} / N_{0}=15$. This figure shows that at any value of $\eta$, the BEP, $P_{e}$, decreases as $E_{b} / N_{j}$ increases. Moreover this figure shows that at large values of $E_{b} / N_{j}, P_{e}$ has approximately the same values. It is also shown that as $\eta$ increases $P_{e}$ increases. The lower bound of the BEP, $P_{l b}$, can be obtained from (16), which is the term in the sum corresponding to $m=L$. With the usual assumption that $N_{0}<<N_{j}, P_{l b}$ becomes a tight lower bound since all other terms in (16) take then much smaller values than $P_{l b}$. Therefore the lower bound of $P_{e}$ after ignoring $N_{0}$ with respect to $N_{j}$ becomes

$$
P_{l b}=\eta^{L} Q\left(\sqrt{\frac{2 \eta E_{b}}{N_{j}}}\right)
$$

The objective of a smart jammer is also achieved if $P_{l b}$ is maximized instead of $P_{e}$. This expression of $P_{l b}$ shows that the worst case parameter $\eta$ depends on the subband order $L$. The plot of lower bound BEP given by (17) versus $E_{b} / N_{j}$ is illustrated in Fig.4. The figure is plotted for different values of $\eta$, and with $L=8$. This figure shows that at any value of $\eta$, the lower bound BEP decreases as $E_{b} / N_{j}$ increases. The plot of lower bound BEP versus the fraction of bandwidth occupied by the jamming $(\eta)$ is illustrated in Fig.5. The figure is plotted for different values of $E_{b} / N_{j}=(-5,0,5$, and $10 \mathrm{~dB})$ and with $L=4$. This figure shows that the lower bound BEP increases as $\eta$ increases. The plot of lower bound BEP versus the subband order $L$ is shown in Fig.6. The figure is plotted for different values of $E_{b} / N_{j}=(-5,0,5$, and $10 \mathrm{~dB}$ ) and with $\eta=0.75$. This figure shows that the lower bound BEP decreases as $L$ increases.

\subsection{Performance Comparison of MC-FH/BPSK System with Single Carrier FH/BPSK System under Partial Band Jamming.}

In this subsection, the comparison between the performance of MC-FH/BPSK system and the single carrier FH/BPSK is provided. The purpose of this comparison is to show superiority of MC-FH/BPSK system over the single carrier system in presence of partial band jamming. The BEP of single carrier FH/BPSK in the presence of partial band jamming is given by:

$$
P_{e}=Q\left(\sqrt{\frac{2 E_{b}^{\prime}}{N_{0}+J_{0}}}\right)=Q\left(\sqrt{\frac{2 E_{b}^{\prime}}{N_{0}+\frac{N_{j}}{\eta}}}\right)
$$


where $E_{b}^{\prime}$ is the energy per bit for the single carrier system. The comparison between the performance of the single carrier system (eq.18) and the performance of the MCFH/BPSK system (eq.16) is shown in Fig.7. This figure is plotted for $\eta=.75$, $E_{b} / N_{o}=10 \mathrm{~dB}$, and $L=4$. This figure shows that under partial band jamming, the MCFH/BPSK system has superior performance than the single carrier system.

\subsection{Broadband Jamming.}

In a broadband jamming the jammer spreads its total power $J$ uniformly across the total bandwidth $W_{s s}$. The conditional probability of error given $m$ for broadband jamming, $P_{B}(e / m)$, is obtained by substituting $m=L$ in (13). Then, the BEP, $P_{e}$, of the MC-FH/BPSK system in presence of broadband jamming is given by

$$
P_{e}=\sum_{m=0}^{L} P_{m} P(e / m)=Q\left(\sqrt{\frac{2 E_{b}}{N_{0}+N_{j}}}\right)
$$

In (19), $P_{m}$ is the probability that a subband is jammed which is similar for all subbands and it is equal to $1 / L$. Similarly it is easy to deduce the BEP under broad band jamming for single carrier FH/BPSK system which results in

$$
P_{e}=Q\left(\sqrt{\frac{2 E_{b}^{\prime}}{N_{0}+N_{j}}}\right)
$$

The comparison between the performance of MC-FH/BPSK system (eq.19) and the performance of single carrier FH/BPSK system (eq.20) under broadband jamming is shown in Fig.8. This figure is plotted for $E_{b} / N_{0}=10 \mathrm{~dB}$ and $L=4$. it is shown that under broadband jamming the MC-FH/BPSK system has superior performance than the single carrier system.

\section{COMPUTER SIMULATIONS AND RESULTS}

In this section, the performance of the MC-FH/BPSK system is evaluated under different jamming techniques discussed above. The parameters that are used in simulation are as follows. The total spread spectrum bandwidth $W_{s s}=40 \mathrm{kHz}$, the information bit rate $R_{b}=5 \mathrm{kbits} / \mathrm{s}$, subband order $L=4$, the sampling rate $f_{s}=80,000$ sample/s, the total number of samples $n_{s}=4096$, and the total number of bits in frame $n=256$. The experiment is repeated 100 times. The total bandwidth is divided into four equal subbands with frequency ranges as illustrated in Table 1. 
Table 1: The frequency range of each subband

\begin{tabular}{|c|c|}
\hline Subband order (L) & Frequency range \\
\hline$L 1$ & $0-10 \mathrm{kHz}$ \\
\hline$L 2$ & $10-20 \mathrm{kHz}$ \\
\hline$L 3$ & $20-30 \mathrm{kHz}$ \\
\hline$L 4$ & $30-40 \mathrm{kHz}$ \\
\hline
\end{tabular}

First, the performance of MC-FH/BPSK system under the effect of partial band jamming is evaluated. Four filters are used to control the bandwidth of the jamming power in each subband. The partial jamming power density, denoted by $J_{0}$, is calculated as $J_{0}=N_{j} / \eta$, where $\eta$ is the ratio of the bandwidth of the filter to total SS bandwidth of the four subbands of the system. The curve of the theoretical and simulated MC-FH/BPSK system under partial band jamming is shown in Fig. 9, The curve is plotted for $\eta=0.75$. This figure shows that there is a slight difference between the theoretical and the simulated results. The reason for this difference is that the filter that used to limit the jamming power is not ideal. Now, the performance of MC-FH/BPSK system under the effect of broadband jamming is examined. The broadband jamming is represented by a Gaussian noise. The signal to jamming ratio is defined as $10 \log \left(E_{b} / N_{j}\right)$. The results of the simulation along with the theoretical results are illustrated in Fig.10. The figure is plotted for $E_{b} / N_{0}=10 \mathrm{~dB}$. This figure shows that the simulation results agree with the theoretical results.

\section{CONCLUSIONS}

The performance of MC-FH/BPSK system under the effect of jamming has been studied. The considered types of jamming are partial band jamming and broadband jamming. Theoretical performance evaluation of the system under both types of jamming has been derived. Computer simulations have been performed to validate the theoretical developments. It has been shown that worst case jamming is difficult to be obtained for this system because it would imply the presence of a jammer in all subbands. 


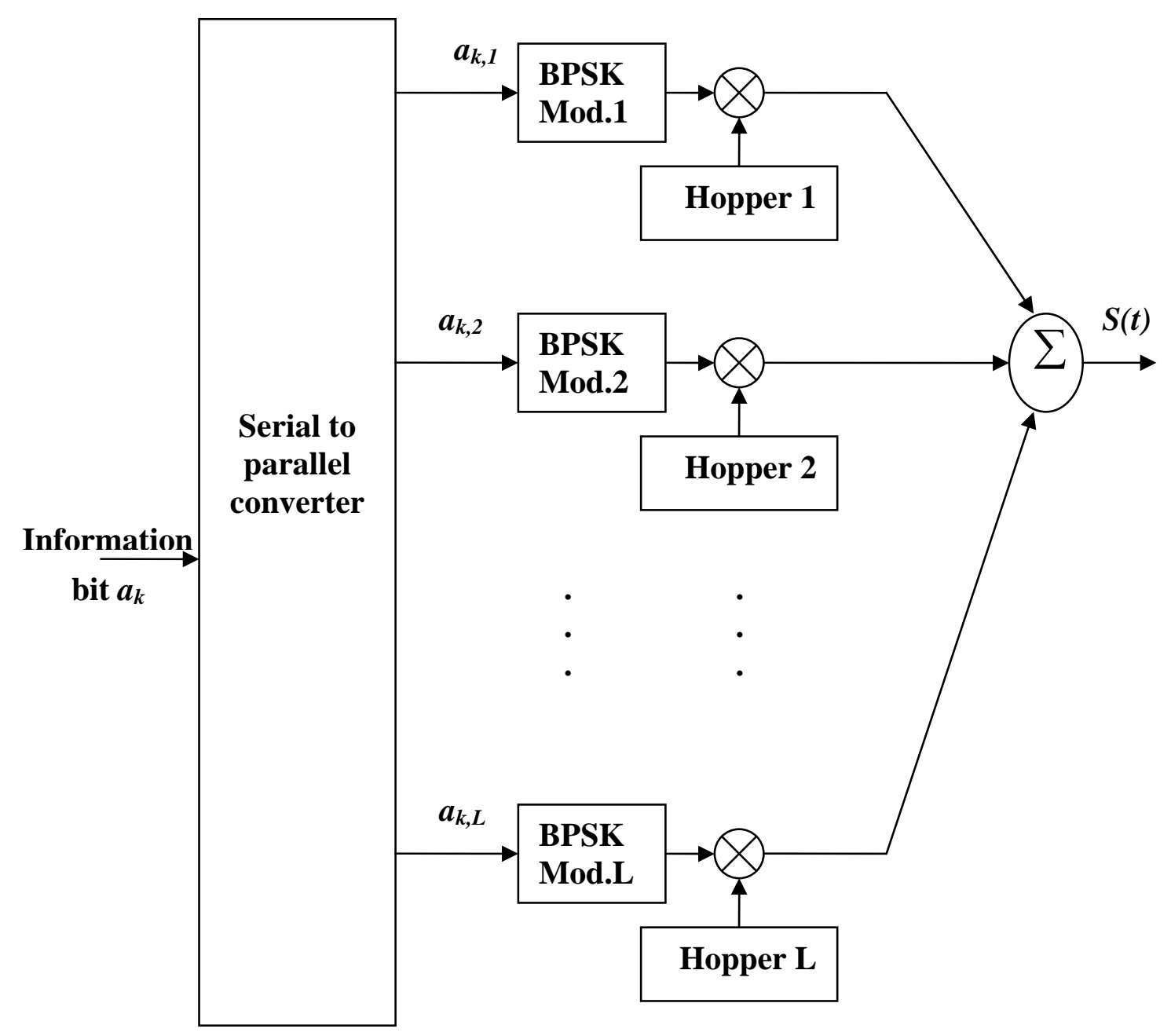

Fig.1. Block diagram of the MC-FH transmitter . 


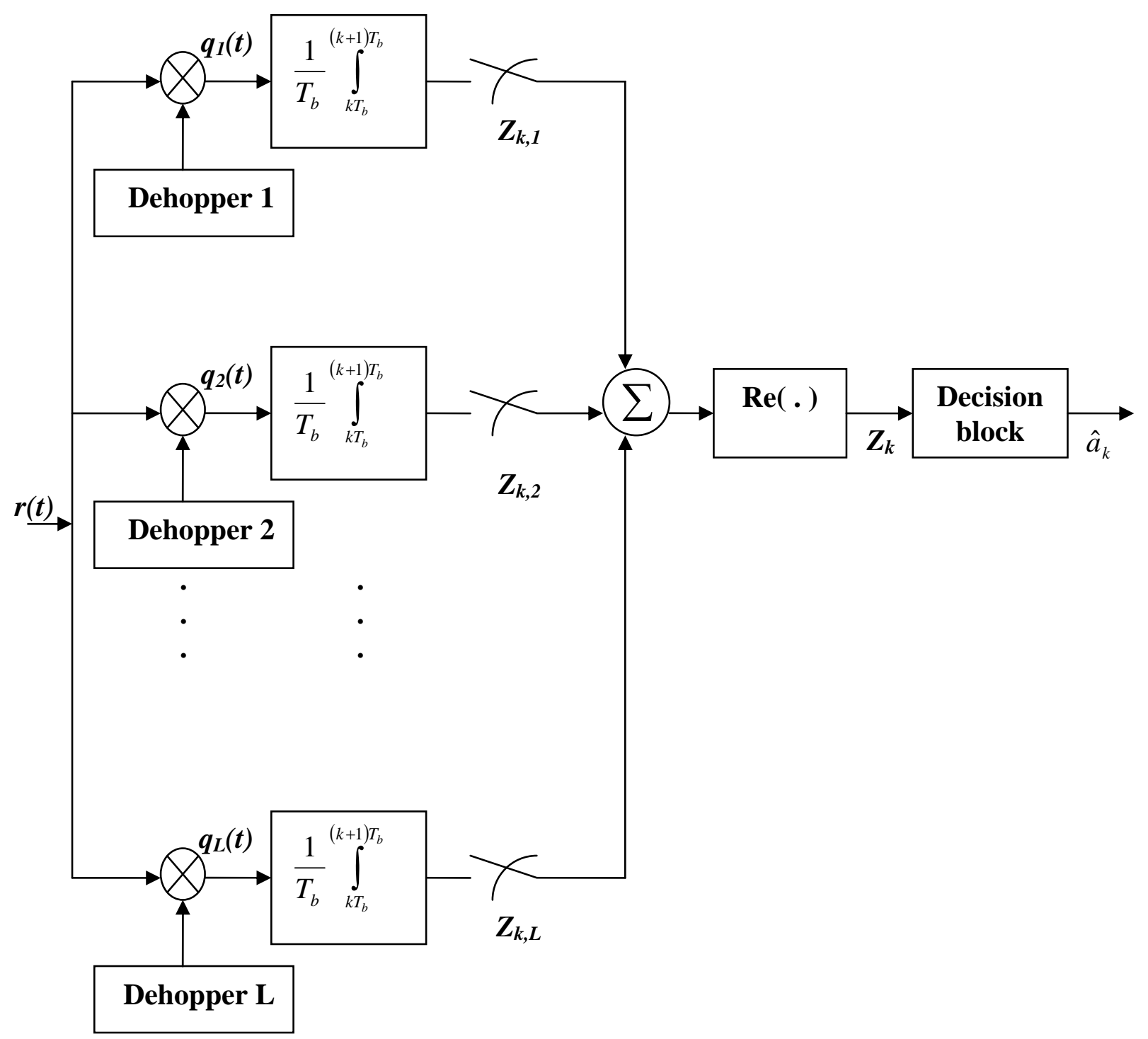

Fig.2. Block diagram of the MC-FH receiver. 


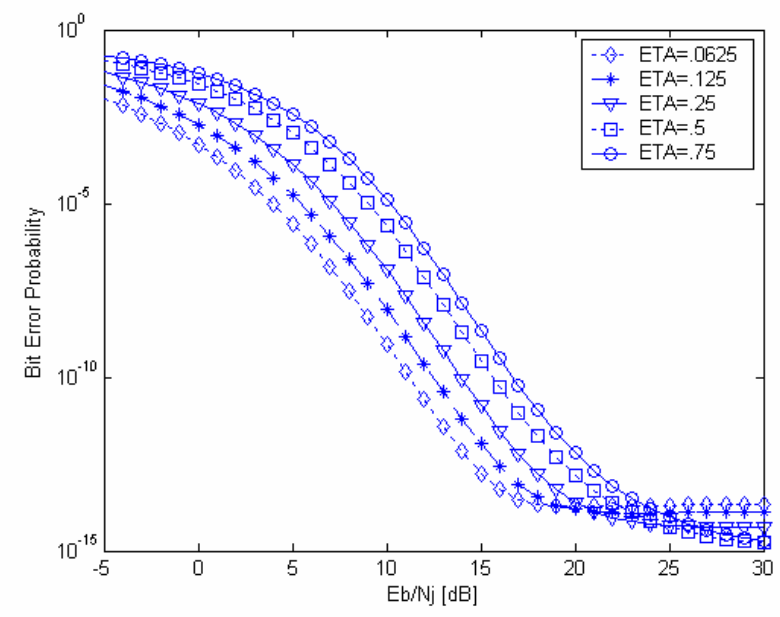

Fig.3. Exact probability of error at $L=M=8$, and $E_{b} / N_{0}=15 \mathrm{~dB}$

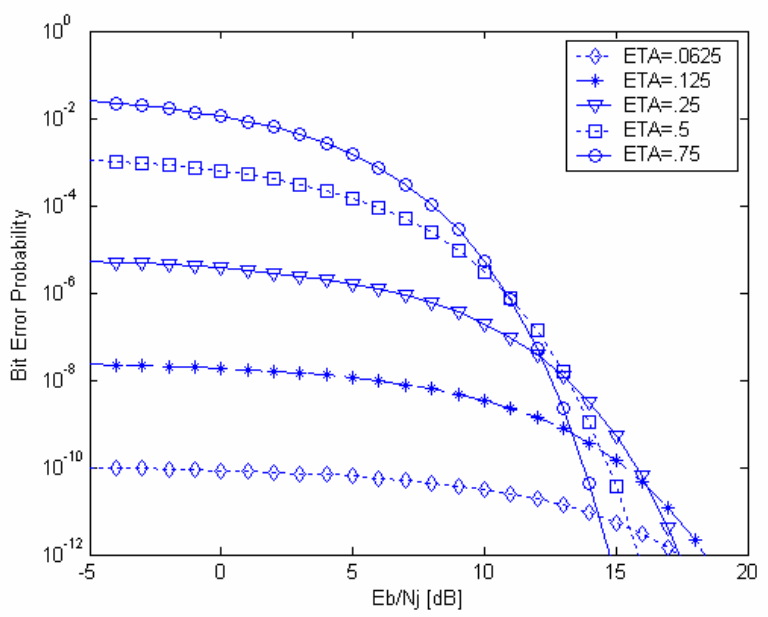

Fig.4. Lower bound of the bit error probability at $L=8$.

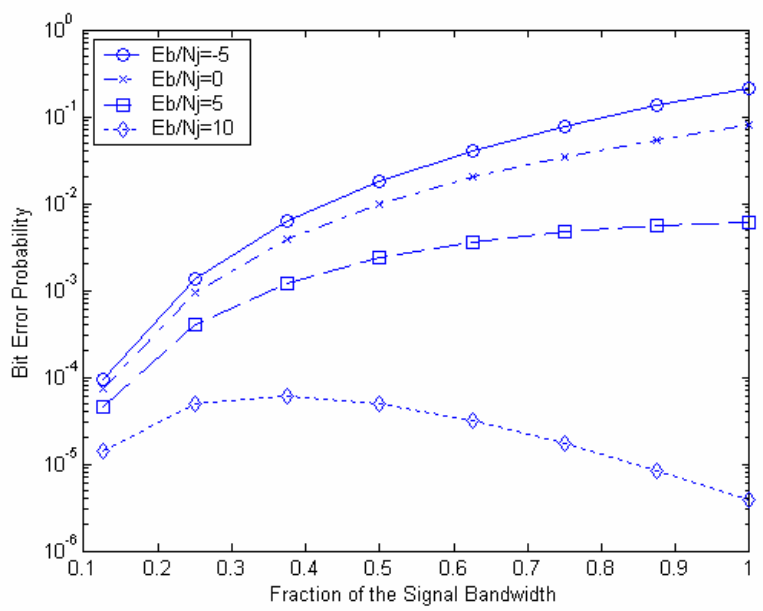

Fig.5. Lower bound of BEP versus $\eta$ for MC-FH/BPSK . 


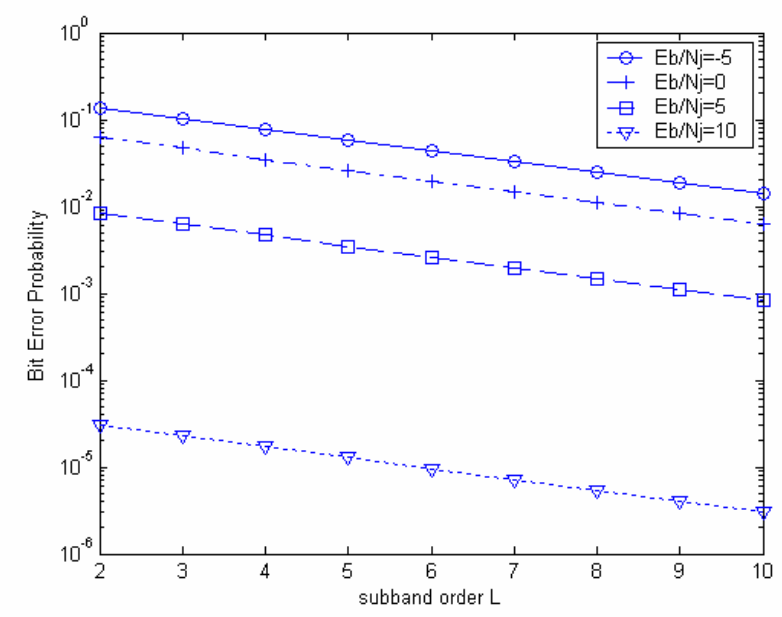

Fig.6. Lower bound of BEP versus $L$ for MC-FH/BPSK

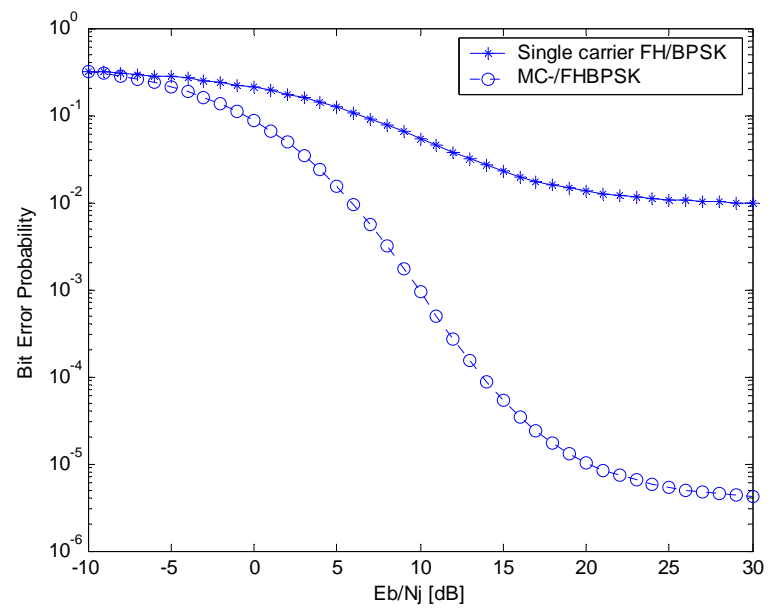

Fig.7. Performance comparison of MC-FH/BPSK and FH/BPSK in presence of partial band jamming at $L=4, \eta=.75$, and $E_{b} / N_{0}=10$ [dB].

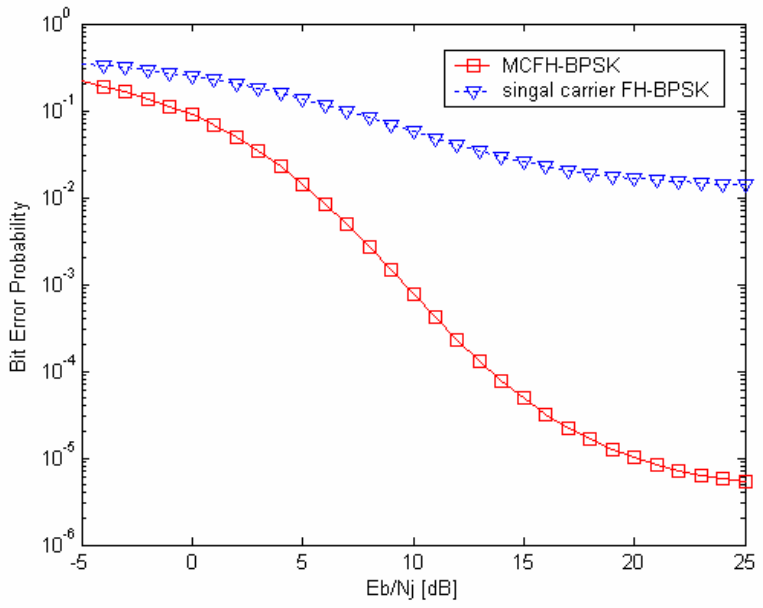

Fig.8. Performance comparison of MC-FH/BPSK and FH/BPSK under broadband jamming for $L=4$ and $E_{b} / N_{0}=10[\mathrm{~dB}]$. 


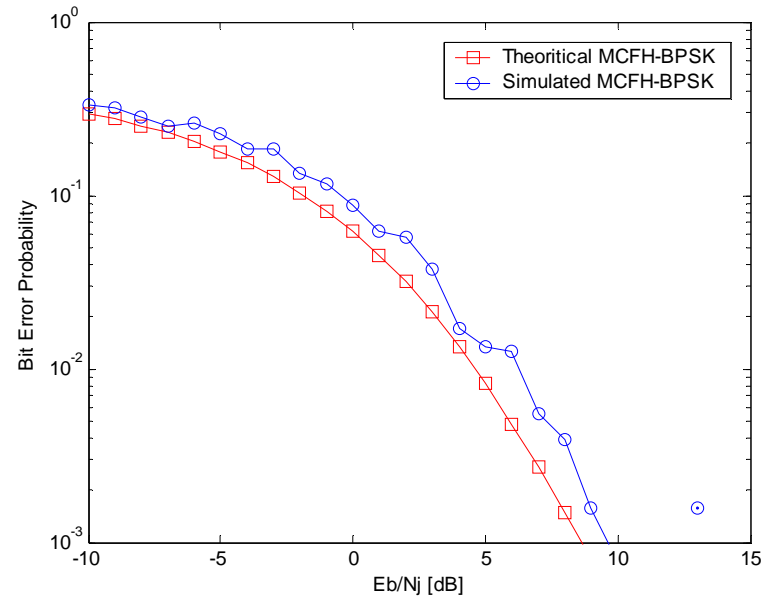

Fig.10. Performance comparison of Simulated and theoretical MC-FH/BPSK under partial band jamming at $L=4$ and $\eta=.75$.

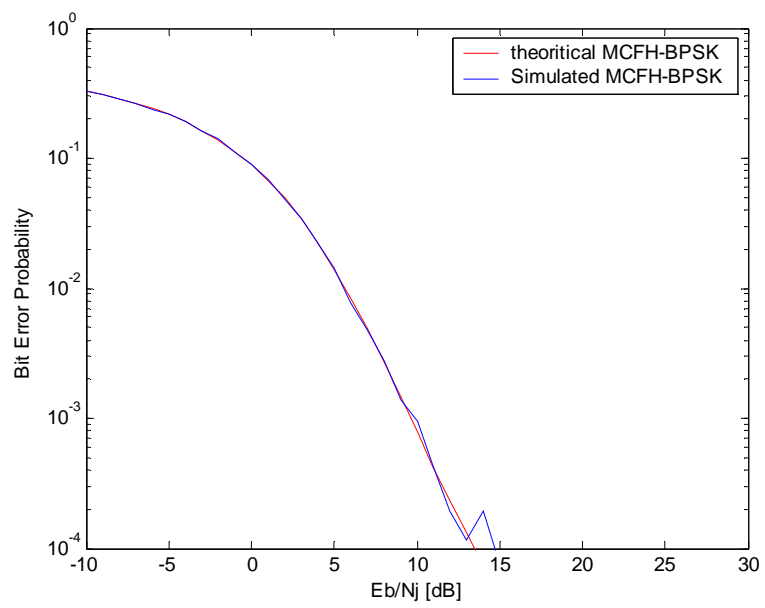

Fig.11. Performance comparison of Simulated and theoretical MC-FH/BPSK under broadband jamming at $L=4$. 


\section{REFERENCES}

[1] Kang, J.J. and Teh, K.C.; Performance of coherent fast frequency-hopped spreadspectrum receivers with partial-band noise jamming and AWGN", IEE proc.Commun., vol 152, No. 5, Oct. 2005 pp:679 - 685.

[2] Chung-Liang Chang; Te-Ming Tu; "Performance analysis of FFH/BFSK productcombining receiver with partial-band jamming over independent Rician fading channels", IEEE Trans. on Wireless commun., vol 4, No.6, Nov. 2005 pp:2629 2635

[3] Jhih, J. L., Li, D. J. and Chung, H. W., "A New partial band noise jamming model for frequency-hopped MFSK systems," IEEE International Conf., pp. 200-204, Sept. 2005.

[4] I. Ghareeb and A. Yonga, coglu, "Performance analysis of frequency hopped/coherent MPSK in the presence of multitone jamming," IEEE Trans.Commun., vol. 44, pp. 152-155, Feb. 1996.

[5] C. M. Su and L. B. Milstein, "Analysis of coherent frequency-hopped spreadspectrum receiver in the presence of jamming," IEEE Trans. Commun., vol. 38, pp.715-726, May 1990.

[6] O.S. Shin and K. B. Lee, "Performance comparison of FFH and MCFH spreadspectrum systems with optimum diversity combining in frequency-selective Rayleigh fading channels," IEEE Trans. Commun., vol. 49, pp. 409-416, March 2001. 